\title{
On two problems of Mordell about exponential sums
}

\author{
by
}

\section{Hong Bing Yu (Hefei)}

1. Introduction. In his last papers, Mordell $([2,3])$ considered a new type of exponential sums and propounded several interesting problems, two of which we shall discuss in the present note.

Throughout, $p$ is an odd prime, $g$ (and $g_{1}$ ) are primitive roots $\bmod p$, $1 \leq X \leq p-1$, and $e_{r}(x)=\exp (2 \pi i x / r)$ as usual.

The first problem suggested by Mordell (see [2]) is to estimate

$$
S_{1}=\sum_{x=1}^{X} e_{p}\left(a x+b g^{x}+c g_{1}^{x}\right), \quad a b c \not \equiv 0(\bmod p),
$$

which is an associated exponential sum of

$$
S_{0}=\sum_{x=1}^{X} e_{p}\left(a x+b g^{x}\right), \quad a b \not \equiv 0(\bmod p) .
$$

In [2] Mordell proved that

$$
\left|S_{0}\right| \leq 2 \sqrt{p} \log p+2 \sqrt{p}+1
$$

he also remarked that the method he used does not appear to be applicable to $S_{1}$. We shall prove

Theorem 1. Let $d=\min \left(\operatorname{ind}_{g} g_{1}, \operatorname{ind}_{g_{1}} g\right), d>1$. Then

$$
\left|S_{1}\right| \leq d^{1 / 4} p^{3 / 4}(2 \log p+3) \text {. }
$$

The second problem relates to

$$
S_{n}(X, b)=\sum_{x=1}^{X} e_{p}\left(b x+f_{n}\left(g^{x}\right)\right)
$$

where $b \not \equiv 0(\bmod p)$, and

(4) $\quad f_{n}(x)=a_{n} x^{n}+\ldots+a_{1} x \in \mathbb{Z}[x], \quad a_{n} \not \equiv 0(\bmod p), n<p$.

1991 Mathematics Subject Classification: Primary 11L07.

Project supported by the National Natural Science Foundation of China. 
Mordell [3] proved, by using an elementary argument, that

$$
\left|S_{n}(p-1, b)\right| \ll p^{1-1 /(2 n)}
$$

where the implied constant depends only on $n$. Further he asked whether $1 / 2$ is the best possible value of the exponent in (5). The following Theorem 2 answers this question affirmatively.

THEOREM 2. We have

$$
\left|S_{n}(X, b)\right| \leq n \sqrt{p}(2 \log p+3) ;
$$

and, for $X>8 n^{2} \log ^{2} p$,

$$
\max _{1 \leq b \leq p-1}\left|S_{n}(X, b)\right| \geq \sqrt{X / 2} .
$$

Theorem 2 is easily generalized. We have

TheOREM 3. Let $f_{n}(x)$ be as in (4), and let

$$
h_{m}(x)=b_{m} x^{m}+\ldots+b_{1} x \in \mathbb{Z}[x], \quad b_{m} \not \equiv 0(\bmod p), m<p .
$$

Write

$$
S_{m, n}(X)=\sum_{x=1}^{X} e_{p}\left(h_{m}(x)+f_{n}\left(g^{x}\right)\right) .
$$

Then

$$
\left|S_{m, n}(X)\right| \leq 4 p^{1-1 / 2^{m}}(n \log p)^{1 / 2^{m-1}} .
$$

By Theorem 3, (13) (below) and Weyl's criterion we immediately have the following result, which may be of independent interest.

COROLlaRY. For any fixed $f_{n}(x)$ satisfying (4) and an arbitrary $h_{m}(x) \in$ $\mathbb{Z}[x]$, the numbers $h_{m}(x)+f_{n}\left(g^{x}\right)$ are uniformly distributed modulo $p$ for $1 \leq x \leq p$, when $p$ is sufficiently large.

It should be mentioned here that, in different contexts, the exponential sums (8) (and hence (1), (2) and (3)) have been generalized by Niederreiter (see Lidl and Niederreiter [1, Chapter 8, §7]). However, his results do not imply ours.

2. The proof of Theorems 1 and 2. To prove Theorem 1 we need the following lemma.

Lemma 1. Let $\chi$ be a Dirichlet character $(\bmod p), b, c$ and $d$ be integers with $b c \neq \equiv 0(\bmod p), d>1$ and $(p-1, d)=1$. Write

$$
S_{\chi}(b, c)=\sum_{x=1}^{p-1} \chi(x) e_{p}\left(b x+c x^{d}\right) .
$$


Then

$$
\left|S_{\chi}(b, c)\right| \leq d^{1 / 4} p^{3 / 4} .
$$

Proof. This can be proved by a well-known method due to Mordell. It is easily seen that

$$
\sum_{u=0}^{p-1} \sum_{v=0}^{p-1}\left|S_{\chi}(u, v)\right|^{4} \leq p^{2} \sum_{s=0}^{p-1} \sum_{t=0}^{p-1} N^{2}(s, t)
$$

where $N(s, t)$ denotes the number of solutions of the congruences

$$
\left\{\begin{array}{l}
x+y \equiv s(\bmod p) \\
x^{d}+y^{d} \equiv t(\bmod p) .
\end{array}\right.
$$

Since $d$ is odd, it follows that $N(0,0)=p, N(s, t)=0$ when only one of $s, t$ is zero and $N(s, t) \leq d-1$ when $s t \neq 0$. Hence the right hand side of $(9)$ is

$$
\begin{aligned}
& \leq p^{2}\left(N^{2}(0,0)+(d-1) \sum_{s, t=1}^{p-1} N(s, t)\right) \\
& \leq p^{2}\left(p^{2}+(d-1)(p-1)(p-2)\right) \leq p^{3}(p-1) d .
\end{aligned}
$$

On the other hand, for any $k \not \equiv 0(\bmod p)$, we have $\left|S_{\chi}(b, c)\right|=\left|S_{\chi}\left(b k, c k^{d}\right)\right|$. Also, for given $u, v$, the congruences

$$
\left\{\begin{array}{l}
b k \equiv u(\bmod p) \\
c k^{d} \equiv v(\bmod p)
\end{array}\right.
$$

have at most one solution in $k$. Hence

$$
\left|S_{\chi}(b, c)\right|^{4}=\frac{1}{p-1} \sum_{k=1}^{p-1}\left|S_{\chi}\left(b k, c k^{d}\right)\right|^{4} \leq \frac{1}{p-1} \sum_{u=0}^{p-1} \sum_{v=0}^{p-1}\left|S_{\chi}(u, v)\right|^{4} \leq p^{3} d,
$$

as required.

Proof of Theorem 1. We may assume without loss of generality that $d=\operatorname{ind}_{g} g_{1}$. By the finite Fourier expansion of $e_{p}\left(b g^{x}+c g^{d x}\right)$, we have, for $x=1, \ldots, X$,

$$
e_{p}\left(b g^{x}+c g^{d x}\right)=\sum_{k=1}^{p-1} c_{k} e_{p-1}(k x),
$$

where the Fourier coefficients $c_{k}$ are given by the formula

$$
c_{k}=\frac{1}{p-1} \sum_{y=1}^{p-1} e_{p}\left(b g^{y}+c g^{d y}\right) e_{p-1}(-k y), \quad k=1, \ldots, p-1 .
$$

By Lemma 1 (setting $\chi(x)=e_{p-1}\left(-k \operatorname{ind}_{g} x\right)$ and $\left.d=\operatorname{ind}_{g} g_{1}\right)$ we have

$$
\left|c_{k}\right| \leq \frac{1}{p-1} d^{1 / 4} p^{3 / 4} \quad \text { for } k=1, \ldots, p-1 .
$$


Thus, by (1) and (10) (noting that $\left.g_{1}^{x} \equiv g^{d x}(\bmod p)\right)$, we get

$$
S_{1}=\sum_{x=1}^{X} \sum_{k=1}^{p-1} c_{k} e_{p-1}(k x) e_{p}(a x)=\sum_{k=1}^{p-1} c_{k} \sum_{x=1}^{X} e_{p-1}(k x) e_{p}(a x) .
$$

From this and (11), we have

$$
\left|S_{1}\right| \leq \frac{1}{p-1} d^{1 / 4} p^{3 / 4} \sum_{k=1}^{p-2} \frac{1}{\left|\sin \left(\frac{a}{p}+\frac{k}{p-1}\right) \pi\right|}+3 \frac{d^{1 / 4} p^{3 / 4}}{p-1} X,
$$

where the accent indicates that two values of $k$, to be chosen the same as in Mordell [2, pp. 86-87], are omitted from the summation (cf. [2, (8)]). Then, by the estimate in [2], we have

$$
\left|S_{1}\right| \leq 2 d^{1 / 4} p^{3 / 4} \log p+3 d^{1 / 4} p^{3 / 4}=d^{1 / 4} p^{3 / 4}(2 \log p+3) .
$$

This proves Theorem 1 .

Proof of Theorem 2. We first prove (6), which is in fact a consequence of Weil's bounds on exponential sums and hybrid sums.

In analogy to (10), we have, for $x=1, \ldots, X$,

$$
e_{p}\left(f_{n}\left(g^{x}\right)\right)=\sum_{k=1}^{p-1} c_{k}^{\prime} e_{p-1}(k x)
$$

where the $c_{k}^{\prime}$ are given by

$$
c_{k}^{\prime}=\frac{1}{p-1} \sum_{y=1}^{p-1} e_{p}\left(f_{n}\left(g^{y}\right)\right) e_{p-1}(-k y), \quad k=1, \ldots, p-1 .
$$

By Weil's bounds (see Schmidt [4, Corollary II.2F and Theorem II.2G]), we have

$$
\left|c_{k}^{\prime}\right| \leq \frac{n \sqrt{p}}{p-1}, \quad k=1, \ldots, p-1 .
$$

Then, similar to the above,

$$
\left|S_{n}(X, b)\right|=\left|\sum_{k=1}^{p-1} c_{k}^{\prime} \sum_{x=1}^{X} e_{p-1}(k x) e_{p}(b x)\right| \leq 2 n \sqrt{p} \log p+3 n \sqrt{p}
$$

as required.

To prove (7), we note that

$$
\sum_{b=0}^{p-1}\left|S_{n}(X, b)\right|^{2}=\sum_{x, y=1}^{X} \sum_{b=0}^{p-1} e_{p}\left(b(x-y)+f_{n}\left(g^{x}\right)-f_{n}\left(g^{y}\right)\right)=p X .
$$


Moreover, from Weil's bounds mentioned above, it is easily seen that

$$
\left|\sum_{x=1}^{X} e_{p}\left(f_{n}\left(g^{x}\right)\right)\right| \leq 2 n \sqrt{p} \log p .
$$

This together with (12) gives (7) at once.

3. The proof of Theorem 3. We require the lemma below.

Lemma 2. Let $F(x)$ be an arbitrary function, and let $\Delta_{h} F(x)=$ $F(x+h)-F(x)$. Then

$$
\left|\sum_{x=1}^{Y} e(F(x))\right|^{2}=Y+\sum_{r=1}^{Y-1} \sum_{y=1}^{Y-r} e\left(\Delta_{r} F(y)\right)+\sum_{r=1}^{Y-1} \sum_{y=Y+1-r}^{Y} e\left(\Delta_{r-Y} F(y)\right),
$$

where $Y$ is a positive integer and $e(u)=\exp (2 \pi i u)$.

Proof. We have

$$
\left|\sum_{x=1}^{Y} e(F(x))\right|^{2}=Y+\sum_{\substack{x, y=1 \\ x \neq y}}^{Y} e(F(x)-F(y)) .
$$

When $x \neq y, 1 \leq|x-y| \leq Y-1$. For any $r(1 \leq r \leq Y-1)$, the solutions of $x-y=r$ are given by $1 \leq y \leq Y-r$; and the solutions of $x-y=-Y+r$ are given by $Y+1-r \leq y \leq Y$. The lemma then follows from (14).

To prove Theorem 3, we proceed by induction on $m$. When $m=1$ the result follows from Theorem 2. Assume that Theorem 3 is true with $m$ replaced by $m-1(m \geq 2)$. By Lemma 2 , we have

$$
\begin{aligned}
\left|S_{m, n}(X)\right|^{2}= & X+\sum_{r=1}^{X-1} \sum_{y=1}^{X-r} e_{p}\left(\Delta_{r} h_{m}(y)+\Delta_{r} f_{n}\left(g^{y}\right)\right) \\
& +\sum_{r=1}^{X-1} \sum_{y=X+1-r}^{X} e_{p}\left(\Delta_{r-X} h_{m}(y)+\Delta_{r-X} f_{n}\left(g^{y}\right)\right) .
\end{aligned}
$$

Write $T(r)$ for the inner sum of the first double sum in (15). Note that

$$
\Delta_{r}\left(f_{n}\left(g^{y}\right)\right)=a_{1}\left(g^{r}-1\right) g^{y}+\ldots+a_{n}\left(g^{n r}-1\right) g^{n y} .
$$

Let $a_{k_{s}}(1 \leq s \leq t \leq n)$ be all those $a_{i}$ such that $a_{k_{s}} \not \equiv 0(\bmod p)$, and let $l=\left(k_{1}, \ldots, k_{t}\right)$. For $1 \leq r \leq X$, if

$$
g^{k_{s} r} \equiv 1(\bmod p) \quad \text { for } s=1, \ldots, t,
$$

then $(p-1) \mid r l$, and so $\frac{p-1}{(p-1, l)} \mid r$. Thus the number of solutions of $(16)$ is at most $(l, p-1) \leq l \leq n$. For these solutions $r$, obviously $|T(r)| \leq X-r \leq X$. For the remaining $r$ 's, $a_{k_{s}}\left(g^{k_{s} r}-1\right)(1 \leq s \leq t)$ are not all $\equiv 0(\bmod p)$. 
Moreover, $\Delta_{r} h_{m}(y)(\bmod p)$ has degree $m-1$ with respect to $y$. Hence, by the induction hypothesis,

$$
|T(r)| \leq 4 p^{1-1 / 2^{m-1}}(n \log p)^{1 / 2^{m-2}} .
$$

Therefore,

$$
\left|\sum_{r=1}^{X-1} T(r)\right| \leq n X+4 p^{1-1 / 2^{m-1}}(n \log p)^{1 / 2^{m-2}} X .
$$

A similar estimate holds for the second double sum in (15). The result then follows easily.

Acknowledgements. The author thanks the referee for the careful reading of the manuscript and useful suggestions.

\section{References}

[1] R. Lidl and H. Niederreiter, Finite Fields, Encyclopedia Math. Appl. 20, Addison-Wesley, 1983.

[2] L. J. Mordell, On the exponential sum $\sum_{x=1}^{X} \exp \left(2 \pi i\left(a x+b g^{x}\right) / p\right)$, Mathematika 19 (1972), 84-87.

[3] -, A new type of exponential series, Quart. J. Math. Oxford Ser. (2) 23 (1972), 373-374.

[4] W. M. Schmidt, Equations over Finite Fields: An Elementary Approach, Lecture Notes in Math. 536, Springer, 1976.

Department of Mathematics

University of Science and Technology of China

Hefei, 230026, Anhui

The People's Republic of China

E-mail: yuhb@math.ustc.edu.cn

Received on 28.7.1997

and in revised form on 5.5.1998 